\title{
ROUGH SET THEORY FOR INTELLIGENT INDUSTRIAL APPLICATIONS
}

\author{
Zdzisław Pawlak
}

Institute of Theoretical and Applied Informatics, Polish Academy of Sciences, Poland, e-mail: zpw@ii.pw.edu.pl

\begin{abstract}
Application of intelligent methods in industry becomes a very challenging issue nowadays and will be of extreme importance in the future. Intelligent methods include fuzzy sets, neural networks, genetics algorithms and others techniques known as soft computing. No doubt rough set theory can also contribute essentially to this domain. In this paper basic ideas of rough set theory are presented and some possible intelligent industrial applications outlined.
\end{abstract}

\section{INTRODUCTION}

Rough set theory is a new mathematical approach to data analysis. Basic idea of this method hinges on classification of objects of interest into similarity classes (clusters) containing objects which are indiscernible with respects to some features, e.g. color, temperature, etc. which form basic building blocks of knowledge about reality and are employed to find out hidden patterns in data. Basis of rough set theory can be found in [20, 22, 26].

Rough set theory has some overlaps with other methods of data analysis, e.g. statistics, cluster analysis, fuzzy sets, evidence theory and others but it can be viewed in its own rights as an independent discipline.

The rough set approach seems to be of fundamental importance to AI and cognitive sciences, especially in the areas of machine learning, knowledge acquisition, decision analysis, knowledge discovery from databases, expert systems, inductive reasoning and pattern recognition. It seems particularly important to decision support systems and data mining.

Rough set theory has been successfully applied to solve many real life problems in medicine, pharmacology, engineering, banking, financial and market analysis and others. More about applications of rough set theory can be found in $[9,17,19,26,28,35,40]$ and others.

Very promising new areas of applications of the rough set concept seem to emerge in the near future. They include rough control, rough data bases, rough information retrieval, rough neural networks and others.

\section{ROUGH SETS AND INTELLIGENT INDUSTRIAL APPLICATIONS}

Artificial intelligence approach to industrial processes is real challenge for industry in the years to come. Rough set theory seems to be particularly suited for problem solving in this area. Some examples are briefly disused below.

1) Material sciences. Application of rough sets to new materials design and investigating material properties has already shown the usefulness of the theory in this area. Pioneer work in this domain is due to Jackson et al $[4,5,6]$. It is interesting also to mention the works on application of rough sets to investigation of the relationship between structure and activity of drugs [28]. The method used here can be also applied not only in the case of drugs but for any other kind of materials.

2) Intelligent control. Industrial process control in many cases, especially in the case of highly nonlinear systems, cannot be successfully treated with classical control theory methods. It turned out that in this case fuzzy sets, neural networks, genetic algorithms offer very good solutions. Also rough sets can be used here in many cases. Cement kiln control algorithms obtained from observation of stoker actions and blast furnace control in iron and steel works are exemplary applications of rough set techniques in intelligent industrial control [11]. Satellite attitude control [25] is another non trivial example of the application of rough set theory in intelligent control. More on applications of this theory in control can be found in $[3,8,12,13,17,23,33,34$, $39,41,42,43]$. Rough set approach in control offers simple and fast algorithms, which can be obtained either from observation of the controlled process or mathematical model of the process or a knowledgeable expert.

3) Decision support systems. Rough set based decision support systems can be widely used in many kinds of industrial decision making on various levels, stretching down from specific industrial processes up to management and business decisions [28, 29, 30].

4) Machine diagnosis. Rough set approach has been used to technical diagnosis of mechanical objects using vibroacoustics symptoms [14, 15,16, 31, 32]. 
5) Neural networks. Neural networks have found many interesting applications in intelligent control of industrial processes. Combining neural networks with fuzzy sets adds new dimension to this domain. Rough sets and neural networks can be also linked together and give better results and greater speed than the classical neural network approach alone. Besides, an interesting idea of rough neural network has been proposed in [10]. More about rough sets and neural networks can be found in references given in [26].

6) Varia. Beside the above said domains of intelligent industrial applications of rough sets there are many other fields where rough set approach can be useful [1, 2, 7, 19, 26, 35, 37, 38, 43].

The above discussed list of possible applications of rough sets is of course not exhaustive but shows areas where application of rough set has already proved to be of use.

Rough set approach shows many advantages. The most important ones are listed below.

- Provides efficient algorithms for finding hidden patterns in data.

- Identifies relationships that would not be found while using statistical methods.

- Allows both qualitative and quantitative data.

- Finds minimal sets of data (data reduction).

- Evaluates significance of data.

- Generates sets of decision rules from data.

- It is easy to understand.

- Offers straightforward interpretation of obtained results.

No doubt rough set theory can be very useful in many branches of intelligent industrial applications as an independent, complementary approach or combined together with other areas of soft computing, e.g. fuzzy sets, neural networks, etc.

\section{APPROXIMATIONS - BASIC CONCEPTS OF ROUGH SET THEORY}

Data are usually given in a form of a data table, called also attribute-value table, information table or database. A database is a table, rows of which are labeled by objects, whereas columns are labeled by attributes. Entries of the table are attribute values. An example of a database is shown in Table 1 .

\begin{tabular}{|c|c|c|c|c|}
\hline Store & $E$ & $Q$ & $L$ & $P$ \\
\hline 1 & high & good & no & profit \\
2 & med. & good & no & loss \\
3 & med. & good & no & profit \\
4 & no & avg. & no & loss \\
5 & med. & avg. & yes & loss \\
6 & high & avg. & yes & profit \\
\hline
\end{tabular}

\section{Table 1}

In the database six stores are characterized by four attributes:

$E$ - empowerment of sales personnel,

$Q$ - perceived quality of merchandises,

$L$ - high traffic location,

$P$ - store profit or loss.

Each subset of attributes in the database determines a partition of all objects into clusters having the same attribute values, i.e. displaying the same features expressed in terms of attribute values. In other words all objects revealing the same features are indiscernible (similar) in view of the available information and they form blocks, which can be understood as elementary granules of knowledge. These granules are called elementary sets or concepts, and can be considered as elementary building blocks (atoms) of our knowledge about reality we are interested in. Elementary concepts can be combined into compound concepts, i.e. concepts that are uniquely defined in terms of elementary concepts. Any union of elementary sets is called a crisp set, and any other sets are referred to as rough (vague, imprecise) ones. With every set $X$ we can associate two crisp sets, called the lower and the upper approximation of $X$. The lower approximation of $X$ is the union of all elementary sets which are included in $X$, whereas the upper approximation of $X$ is the union of all elementary sets which have non empty intersection with $X$. In other words the lower approximation of a set is the set of all elements that surely belong to $X$, whereas the upper approximation of $X$ is the set of all elements that possibly belong to $X$. The difference of the upper and the lower approximation of $X$ is its boundary region. Obviously a set is rough if it has non empty boundary region whatsoever; otherwise the set is crisp. Elements of the boundary region cannot be classified employing the available knowledge, either to the set or its complement. Approximations of sets are basic operations in rough set theory and are used as main tools to deal with vague and uncertain data. Let us illustrate the above ideas by means of data given in Table 1.

Each store has different description in terms of attributes $E, Q, L$ and $P$, thus all stores are discernible when employing information provided by all attributes. However, stores 2 and 3 are indiscernible in terms of attributes $E, Q$ and $L$, since they have the same values of these attributes. Similarly, stores 1,2 and 3 are indiscernible with respect to attributes $Q$ and $L$, etc.

Each subset of attributes determines a partition (classification) of all objects into classes having the same description in terms of these attributes. For example, attributes $Q$ and $L$ aggregate all stores into the following classes $\{1,2,3\},\{4\},\{5,6\}$. Thus, each database determines a family of classification patterns which are used as a basis of further considerations. 
Let us consider the following problem: what are the characteristic features of stores making a profit (or having a loss) in view of information available in Table 1 , i.e. we want to describe set (concept) $\{1,3,6\}$ (or $\{2,4,5\}$ ) in terms of attributes $E, Q$ and $L$. Of course this question cannot be answered uniquely since stores 2 and 3 have the same values of attributes $E, Q$ and $L$, but store 2 makes a profit, whereas store 3 has a loss. Hence in view of information contained in Table 1 , we can say for sure that stores 1 and 6 make a profit, stores 4 and 5 have a loss, whereas stores 2 and 3 cannot be classified as making a profit or having a loss. That is employing attributes $E, Q$ and $L$, we can say that stores 1 and 6 surely make a profit, i.e. surely belong to the set $\{1,3,6\}$, whereas stores $1,2,3$ and 6 possibly make a profit, i.e. possibly belong to the set $\{1,3,6\}$. We will say that the set $\{1,6\}$ is the lower approximation of the set (concept) $\{1,3,6\}$, and the set $\{1,2,3,6\}$ is the upper approximation of the set $\{1,3,6\}$. The set $\{2,3\}$, being the difference between the upper approximation and the lower approximation is referred to as the boundary region of the set $\{1,3$, $6\}$.

Now let us give some formal notations and definitions.

By a database we will understand a pair $S=(U, A)$, where $U$ and $A$, are finite, non empty sets called the universe, and a set of attributes respectively. With every attribute $a \in A$ we associate a set $V_{a}$ of its values, called the domain of $a$. Any subset $B$ of $A$ determines a binary relation $I(B)$ on $U$, which will be called an indiscernibility relation, and is defined as follows:

$(x, y) \in I(B)$ if and only if $a(x)=a(y)$ for every $a \in A$, where $a(x)$ denotes the value of attribute $a$ for element $x$.

It can easily be seen that $I(B)$ is an equivalence relation. The family of all equivalence classes of $I(B)$, i.e. partition determined by $B$, will be denoted by $U / I(B)$, or simple $U / B$; an equivalence class of $I(B)$, i.e. block of the partition $U / B$ containing $x$ will be denoted by $B(x)$.

If $(x, y)$ belongs to $I(B)$ we will say that $x$ and $y$ are $B$-indiscernible. Equivalence classes of the relation $I(B)$ (or blocks of the partition $U / B$ ) are referred to as $B$-elementary sets or B-granules.

The indiscernibility relation will be used next to define two basic operations in rough set theory, which are defined below:

$$
\begin{gathered}
B_{*}(X)=\bigcup_{x \in U}\{B(x): B(x) \subseteq X\}, \\
B^{*}(X)=\bigcup_{x \in U}\{B(x): B(x) \cap X \neq \varnothing\},
\end{gathered}
$$

and are called the B-lower and the B-upper approximation of $X$, respectively.

The set

$$
B N_{B}(X)=B^{*}(X)-B_{*}(X)
$$

will be referred to as the $B$-boundary region of $X$.

If the boundary region of $X$ is the empty set, i.e. $B N_{B}(X)=\varnothing$, then the set $X$ is crisp (exact) with respect to $B$; in the opposite case, i.e. if $B N_{B}(X) \neq \varnothing$, the set $\mathrm{X}$ is referred to as rough (inexact) with respect to $B$.

\section{DEPENDENCY ATTRIBUTES}

Approximations of sets are strictly related with the concept of dependency (total or partial) of attributes.

Suppose that the set of attributes $A$ is partitioned into two disjoint subsets $C$ and $D$ called condition and decision attributes, respectively. Databases with distinguished condition and decision attributes will be referred to as decision tables.

Intuitively, a set of decision attributes $D$ depends totally on a set of condition attributes $C$, denoted $C \Rightarrow D$, if all values of decision attributes are uniquely determined by values of condition attributes. In other words, $D$ depends totally on $C$, if there exists a functional dependency between values of $C$ and $D$.

We will also need a more general concept of dependency of attributes, called the partial dependency of attributes. Partial dependency means that only some values of $D$ are determined by values of $C$.

Formally dependency can be defined in the following way.

Let $C$ and $D$ be subsets of $A$, such that $D \cap C \neq \varnothing$ and $D \cup C=A$.

We will say that $D$ depends on $C$ in a degree $k(0 \leq k \leq 1)$, denoted $C \Rightarrow{ }_{k} D$, if

$$
k=\gamma(C, D)=\sum_{X \in U / D} \frac{\operatorname{card}\left(C_{*}(X)\right)}{\operatorname{card}(U)},
$$

where card $(X)$ is the cardinality of $X$.

If $k=1$ we say that $D$ depends totally on $C$, and if $k<1$, we say that $D$ depends partially (in a degree $k$ ) on $C$.

The coefficient $k$ expresses the ratio of all elements of the universe, which can be properly classified to block of the partition $U / D$ employing attributes $C$ and will be called the degree of the dependency.

For example, the attribute $P$ depends on the set of attributes $\{E, Q, L\}$ in the degree $2 / 3$. That means that only four out of six stores can be exactly identified by means of attributes $E, Q$ and $L$ as having a loss or making a profit.

\section{REDUCTION OF ATTRIBUTES}

We often face a question whether we can remove some data from a database preserving its basic proper- 
ties, that is - whether a table contains some superfluous data.

Let us express this idea more precisely.

Let $C, D \subseteq A$, be sets of condition and decision attributes, respectively. We will say that $C^{\prime} \subseteq C$ is a $D$-reduct (reduct with respect to $D$ ) of $C$, if $C^{\prime}$ is a minimal subset of $C$ such that

$$
\gamma(C, D)=\gamma\left(C^{\prime}, D\right)
$$

Hence any reduct enables us to reduce condition attributes in such a way that the degree of dependency between condition and decision attributes is preserved. In other words reduction of condition attributes gives the minimal number of conditions necessary to make specified decisions.

In the database presented in Table $1\{E, Q\}$ and $\{E, L\}$ are the only two reducts of condition attributes with respect to $P$, i.e. either the set $\{E, Q\}$ or the set $\{E, L\}$ can be used to classify stores instead of the whole set of condition attributes $\{E, Q, L\}$.

For large databases finding reducts on the basis of the definition given before is rather difficult because the definition leads to inefficient algorithms. Therefore more efficient methods of reduct computation have been proposed. For details see references in [26].

\section{DECISION RULES}

Every dependency $C \Rightarrow_{k} D$ can be described by a set of decision rules in the form ,if ... then”, written $\Phi \rightarrow \Psi$, where $\Phi$ and $\Psi$ are logical formulas describing conditions and decisions of the rule respectively, and are built up from elementary formulas (attribute, value) combined together by means of propositional connectives ,and”, „or” and „not” in the standard way.

An example of a decision rule is given below:

$$
\text { if (E,med.) and (Q, good) and (L,no) then (P,loss). }
$$

With every decision rule $\Phi \rightarrow \Psi$ we associate conditional probability that $\Psi$ is true in $S$, given $\Phi$ is true in $S$ with the probability $\pi_{S}(\Phi)$ called a certainty factor and defined as follows:

$$
\pi_{S}(\Psi \mid \Phi)=\frac{\operatorname{card}\left(|\Phi \wedge \Psi|_{S}\right)}{\operatorname{card}\left(|\Phi|_{S}\right)},
$$

where $|\Phi|_{S}$ denotes the set of all objects satisfying $\Phi$ in $S$.

Besides, we will also need a coverage factor [36]

$$
\pi_{S}(\Phi \mid \Psi)=\frac{\operatorname{card}\left(|\Phi \wedge \Psi|_{S}\right)}{\operatorname{card}\left(|\Psi|_{S}\right)},
$$

which is the conditional probability that $\Phi$ is true in $S$, given $\Psi$ is true in $S$ with the probability $\pi_{S}(\Psi)$.
For the decision rule given above the certainty and coverage factors are $1 / 2$ and $1 / 3$, respectively, i.e. the probability that the decision pointed out by the decision rule is correct equals to $1 / 2$ and the rule covers one of the three decisions indicated by the rule.

Let $\left\{\Phi_{i} \rightarrow \Psi\right\}_{n}$ be a set of decision rules such that all conditions $\Phi_{i}$ are pairwise mutually exclusive, i.e. $\left|\Phi_{i} \wedge \Phi_{j}\right|_{S}=\varnothing$, for any $1 \leq i, j \leq n, i \neq j$, and

$$
\sum_{i=1}^{n} \pi_{S}\left(\Phi_{i} \mid \Psi\right)=1
$$

For any decision rule $\Phi \rightarrow \Psi$ the following is true:

$$
\pi_{S}(\Phi \mid \Psi)=\frac{\pi_{S}(\Psi \mid \Phi) \cdot \pi_{S}(\Phi)}{\sum_{i=1}^{n} \pi_{S}\left(\Psi \mid \Phi_{i}\right) \cdot \pi_{S}\left(\Phi_{i}\right)}
$$

The relationship between the certainty factor and the coverage factor, expressed by the formula $(*)$ is the Bayes' theorem. The formula shows that any decision table satisfies Bayes' theorem. This property gives a new dimension to the Baysian reasoning methods and enables to discover relationship in data without referring to prior and posterior probabilities, inherently associated with Bayesian philosophy. The above result is of special value for large databases.

\section{CONCLUSION}

Rough set theory proved to be a very well suited candidate, beside fuzzy sets, neural networks and other soft computing methods, for intelligent industrial applications. Particularly challenging areas of applications of rough sets in industrial environment are material sciences, intelligent control, machine diagnosis and decision support.

Rough set approach has many advantageous features, e.g. it identifies relationships that would not be found using statistical methods, allows both qualitative and quantitative data and offers straightforward interpretation of obtained results

Despite many successful applications of rough sets in industry there are still problems which require further research. In particular development of suitable, widely accessible software dedicated to industrial applications as well as microprocessors based on rough set theory are badly needed.

\section{REFERENCES}

1. An, A. Chan, C., Shan, N., Cercone, N., Ziarko, W.: Applying knowledge discovery to predict water-supply consumption. IEEE Expert 12/4 (1997) $72-78$

2. Arciszewski, T., Ziarko, W.: Inductive learning in civil engineering: rough sets approach. Microcomputers and Civil Engineering 5/1 (1990) 
3. Czogala, E., Mrozek, A., Pawlak, Z.: The idea of rough-fuzzy controller. International Journal of Fuzzy Sets and Systems 72 (1995) 61-63

4. Jackson, A. G., Ohmer, M., Al-Kamhawi, H.: Rough sets analysis of chalcopyrite semiconductor band gap data. In: T. Y. Lin (ed.), The Third International Workshop on Rough Sets and Soft Computing Proceedings (RSSC'94), November 10-12, San Jose State University, San Jose, California, USA (1994) 408-417

5. Jackson, A. G., Leclair, S. R., Ohmer, M. C., Ziarko. W., Al-Kamhwi, H.: Rough sets applied to material data. Acta Metallurgica et Materialia (1996) 4475

6. Jackson, A. G., Pawlak, Z., Leclair, S. R.: Rough set and discovery of new materials. Journal of Alloys and Compounds (to appear)

7. Kowalczyk, W.: Analyzing temporal patterns with rough sets. In: EUFIT-96: The fourth European Congress on Intelligent Techniques and Soft Computing, September 2-5, Aachen (1996) 139143

8. Lin, T. Y.: Fuzzy controllers: an integrated approach based on fuzzy logic, rough sets, and evolutionary computing. In: T. Y. Lin and N. Cercone (eds.), Rough Sets and Data Mining. Analysis for Imprecise Data, Kluwer Academic Publishers, Boston, London, Dordrecht (1997) 123-138

9. Lin, T. Y., Cercone, N.,(eds.): Rough Sets and Data Mining - Analysis of Imperfect Data, Kluwer Academic Publishers, Boston, London, Dordrecht (1997) 430

10. Lingras, P.: Rough neural networks. Sixth International Conferences, Information Procesing and Management of Uncertainty in Knowledge-Based Systems, Proceedings (IPMU'96), Volume II, July 1-5, Grenada (1996) 1445-1450

11. Mrozek, A.: Rough sets in computer implementation of rule-based control of industrial processes. In: R. Slowinski (ed.), Intelligent Decision Support. Handbook of Applications and Advances of the Rough Set Theory. Kluwer Academic Publishers, Boston, London, Dordrecht (1992) 19-31

12. Munakata, T.: Rough control: a perspective. In: T. Y. Lin and N. Cercone (eds.), Rough Sets and Data Mining. Analysis for Imprecise Data. Kluwer Academic Publishers, Boston, London, Dordrecht (1997) 77-88

13. Munakata, T.: Fundamentals of the New Artificial Intelligence. Springer (1998) 231

14. Nowicki R., Slowinski R., Stefanowski J.: Possibilities of appling the rough sets theory to technical diagnostics. In: Proceedings of the IXth $\mathrm{Na}-$ tional Symposium on Vibration Techniques and
Vibroacoustics, December 12-14, AGH University Press, Kraków (1990) 149-152

15. Nowicki, R., Slowinski, R., Stefanowski, J.: Rough sets analysis of diagnostic capacity of vibroacoustic symptoms. Journal of Computers and Mathematics with Applications 24/2 (1992) 109123

16. Nowicki, R., Slowinski, R., Stefanowski, J.: Evaluation of vibroacoustic diagnostic symptoms by means of the rough sets theory. Journal of Computers in Industry 20 (1992) 141-152

17. Oehrn, A.: Rough logic control. In: (Project), Technical Report. Knowledge Systems Group, The Norwegian University of Science and Technology, Trondheim, Norway (1993)

18. E. Orlowska (ed.): Incomplete Information: Rough Set Analysis. Physica-Verlag, Heidelberg (1997)

19. S.K. Pal, A. Skowron (eds.): Fuzzy Sets, Rough Sets and Decision Making Processes.Springer-Verlag, Singapore (in preparation)

20. Pawlak, Z.: Rough Sets - Theoretical Aspects of Reasoning about Data. Kluwer Academic Publishers, Boston, London, Dordrecht (1991) 229.

21. Pawlak, Z.: Rough set theory and its applications to data analysis. Cybernetics and Systems 29 (1998) 661-688

22. Pawlak, Z., Grzymala-Busse, J. W., Slowinski, R., Ziarko, W.: Rough sets. Communication of the ACM 38 (1995) 88-95

23. Pawlak, Z., Munakata, T.: Rough control application of rough set theory to control. Fourth European Congress on Intelligent Techniques and Soft Computing, Proceedings EUFIT'96, Volume I, September 2-5, Germany (1996) 209-218

24. Pawlak, Z.: Reasoning about data - a rough set perspective. In: L. Polkowski, A. Skowron (eds.), Rough Sets and Current Trends in Computing, Lecture Notes in Artificial Intelligence, 1424 Springer, First International Conference, RSCTC' 98, Warsaw, Poland, June, Proceedings, (1998) 25-34

25. Peters, J. F, Ziaei, K, Ramanna, S.: Approximate time rough control: Concepts and application to satellite attitude control. In: L. Polkowski, A. Skowron (eds.), Rough Sets and Current Trends in Computing, Lecture Notes in Artificial Intelligence, 1424 Springer, First International Conference, RSCTC' 98, Warsaw, Poland, June, Proceedings, (1998) 491-498

26. L. Polkowski, A. Skowron (eds.): Rough Sets in Knowledge Discovery, Physica-Verlag, Vol. 1, 2 (1998) 
27. L. Polkowski, A. Skowron (eds.): Rough Sets and Current Trends in Computing, Lecture Notes in Artificial Intelligence, 1424 Springer, First International Conference, RSCTC'98, Warsaw, Poland, June, Proceedings, (1998)

28. Slowinski, R.: Intelligent Decision Support. Handbook of Applications and Advances of the Rough Set Theory. Kluwer Academic Publishers, Boston, London, Dordrecht (1992)

29. Slowinski, R.: Rough set approach to decision analysis. AI Expert 10 (1995) 18-25

30. Slowinski, R.: Rough set theory and its applications to decision aid. Belgian Journal of Operation Research, Special Issue Francoro 35/3-4 (1995) 81-90

31. Slowinski, R., Stefanowski, J., Susmaga, R.: Rough set analysis of attribute dependencies in technical diagnostics. In: S. Tsumoto, S. Kobayashi, T. Yokomori, H. Tanaka and A. Nakamura (eds.), The fourth International Workshop on Rough Sets, Fuzzy Sets, and Machnine Discovery, Proceedings (RS96FD), November 6-8, The University of Tokyo (1996) 284-291

32. Stefanowski, J., Slowinski, R., Nowicki, R.: The rough sets approach to knowledge analysis for classification support in technical diagnostics of mechanical objects. In: F. Belli and F. J. Radermacher (eds.), Industrial \& Engineering Applications of Artificial Intelligence and Expert Systems. Lecture Notes in Economics and Mathematical Systems 604, Springer-Verlag, Berlin (1992) 324-334

33. Szladow, A., Ziarko W.: Adaptive process control using rough sets. Proceedings of the International Conference of Instrument Society of America, ISA/93, Chicago (1993) 1421-1430

34. Szladow, A., Ziarko W.: Application of rough sets theory to process control. Proceedings of Calgary 93 Symposium of Instrument Society of America, Calgary (1993)

35. Tsumoto, S., Kobayashi, S., Yokomori, T., Tanaka, H., Nakamura, A., (eds.): The Fourth Internal Workshop on Rough Sets, Fuzzy Sets and
Machine Discovery, PROCEEDINGS. The University of Tokyo (1996)

36. Tsumoto, S.: Modelling medical diagnostic rules based on rough sets. In: L. Polkowski, A. Skowron (eds.), Rough Sets and Current Trends in Computing, Lecture Notes in Artificial Intelligence, 1424 Springer, First International Conference, RSCTC' 98, Warsaw, Poland, June, Proceedings, (1998) 475-482

37. Wang, P. P., (ed.): Second Annual Joint Conference on Information Sciences, PROCEEDINGS. Wrightsville Beach, North Carolina, USA (1995)

38. Wang, P., (ed.): Joint Conference of Information Sciences, Vol. 3. Rough Sets and Computer Sciences, Duke University (1997)

39. Ziarko, W.: Acquisition of control algorithms from operation data. In: R. Slowinski (ed.), Intelligent Decision Support, Handbook of Applications and Advances of the Rough Set Theory, Kluwer Academic Publishers, Boston, London, Dordrecht (1992) 61-75

40. Ziarko, W., (ed.): Rough Sets, Fuzzy Sets and Knowledge Discovery. Proceedings of the International Workshop on Rough Sets and Knowledge Discovery (RSKD'93), Banff, Alberta, Canada, October 12-15, Springer-Verlag, Berlin (1993)

41. Ziarko, W., Katzberg, J.: Control algorithms acquisition, analysis and reduction: machine learning approach. In: Knowledge-Based Systems Diagnosis, Supervision and Control, Plenum Press, Oxford (1989) 167-178

42. Ziarko, W., Katzberg, J.: Rough sets approach to system modelling and control algorithm acquisition. Proceedings of IEEE WESCANEX 93 Conference, Saskatoon (1993) 154-163

43. Zak J., Stefanowski J.: Determining maintenance activities of motor vehicles using rough sets aproach. In: Proceedings of Euromaintenance'94 Conference, Amsterdam (1994) 39-42 